\title{
Measurement of the Muon Capture Rate in Hydrogen Gas and Determination of the Proton's Pseudoscalar Coupling $g_{P}$
}

\author{
V. A. Andreev, ${ }^{1}$ T. I. Banks, ${ }^{2}$ T. A. Case, ${ }^{2}$ D. B. Chitwood, ${ }^{3}$ S. M. Clayton, ${ }^{3}$ K. M. Crowe, ${ }^{2}$ J. Deutsch, ${ }^{4}$ J. Egger, ${ }^{5}$ \\ S. J. Freedman, ${ }^{2}$ V. A. Ganzha, ${ }^{1}$ T. Gorringe, ${ }^{6}$ F. E. Gray, ${ }^{2}$ D. W. Hertzog, ${ }^{3}$ M. Hildebrandt, ${ }^{5}$ P. Kammel, ${ }^{3, *}$ B. Kiburg, ${ }^{3}$ \\ S. Knaack ${ }^{3}{ }^{3}$ P. A. Kravtsov, ${ }^{1}$ A. G. Krivshich, ${ }^{1}$ B. Lauss, ${ }^{2}$ K. L. Lynch, ${ }^{7}$ E. M. Maev, ${ }^{1}$ O. E. Maev, ${ }^{1}$ F. Mulhauser, ${ }^{3,5}$ \\ C. S. Özben, ${ }^{3}$ C. Petitjean, ${ }^{5}$ G. E. Petrov, ${ }^{1}$ R. Prieels, ${ }^{4}$ G. N. Schapkin, ${ }^{1}$ G. G. Semenchuk, ${ }^{1}$ M. A. Soroka, ${ }^{1}$ V. Tishchenko, ${ }^{6}$ \\ A. A. Vasilyev, ${ }^{1}$ A. A. Vorobyov, ${ }^{1}$ M.E. Vznuzdaev, ${ }^{1}$ and P. Winter ${ }^{3}$
}

(MuCap Collaboration)

\author{
${ }^{1}$ Petersburg Nuclear Physics Institute, Gatchina 188350, Russia \\ ${ }^{2}$ University of California, Berkeley, and LBNL, Berkeley, California 94720, USA \\ ${ }^{3}$ University of Illinois at Urbana-Champaign, Urbana, Illinois 61801, USA \\ ${ }^{4}$ Université Catholique de Louvain, B-1348, Louvain-la-Neuve, Belgium \\ ${ }^{5}$ Paul Scherrer Institute, $\mathrm{CH}-5232$ Villigen PSI, Switzerland \\ ${ }^{6}$ University of Kentucky, Lexington, Kentucky 40506, USA \\ ${ }^{7}$ Boston University, Boston, Massachusetts 02215, USA \\ (Received 16 April 2007; published 16 July 2007)
}

\begin{abstract}
The rate of nuclear muon capture by the proton has been measured using a new technique based on a time projection chamber operating in ultraclean, deuterium-depleted hydrogen gas, which is key to avoiding uncertainties from muonic molecule formation. The capture rate from the hyperfine singlet ground state of the $\mu p$ atom was obtained from the difference between the $\mu^{-}$disappearance rate in hydrogen and the world average for the $\mu^{+}$decay rate, yielding $\Lambda_{S}=725.0 \pm 17.4 \mathrm{~s}^{-1}$, from which the induced pseudoscalar coupling of the nucleon, $g_{P}\left(q^{2}=-0.88 m_{\mu}^{2}\right)=7.3 \pm 1.1$, is extracted.
\end{abstract}

PACS numbers: 13.60.- r, 11.40.Ha, 14.20.Dh, 23.40.-s

We report the first result of the MuCap experiment for the rate $\Lambda_{S}$ of the semileptonic weak process of ordinary muon capture (OMC) by the proton,

$$
\mu^{-}+p \rightarrow n+\nu_{\mu}
$$

This fundamental process, like neutron beta decay, involves the vector and axial-vector form factors $g_{V}\left(q^{2}\right)$ and $g_{A}\left(q^{2}\right)$, which characterize the microscopic QCD structure of the nucleon in electroweak charged-current interactions. Because of its larger momentum transfer $q_{0}^{2}=$ $-0.88 m_{\mu}^{2}$, reaction (1) is also sensitive to the weak magnetic and pseudoscalar induced form factors, $g_{M}\left(q^{2}\right)$ and $g_{P}\left(q^{2}\right)$. Form factors $g_{V}\left(q_{0}^{2}\right), g_{M}\left(q_{0}^{2}\right)$, and $g_{A}\left(q_{0}^{2}\right)$ are accurately determined by experimental data and standard model symmetries and contribute an uncertainty of only $0.46 \%$ to $\Lambda_{S}$ [1]. Process (1) provides the most direct probe of $g_{P} \equiv$ $g_{P}\left(q_{0}^{2}\right)$, the pseudoscalar coupling of the nucleon's axial current, which is by far the least well known of these form factors.

The form factor $g_{P}\left(q^{2}\right)$ arises mainly from the coupling of the weak leptonic current to the nucleon via an intermediate pion, which generates a pole term that dominates at $q_{0}^{2}$. Early theoretical expressions for $g_{P}$ were derived from the concept of the partially conserved axial current; now $g_{P}$ can be systematically calculated within heavy baryon chiral perturbation theory (HBChPT) up to two- loop order [2]. The precise result $g_{P}=8.26 \pm 0.23$ [3] follows from the basic concepts of explicit and spontaneous chiral symmetry breaking, and thus its experimental confirmation is an important test of QCD symmetries [3-5].

Experimental OMC efforts span a period of more than 40 years, and more recently radiative muon capture (RMC) by the proton was measured for the first time [6]. However, as shown in Fig. 1, the situation prior to the present experiment was inconclusive, as the results lacked sufficient precision due to ambiguities in the interpretation as well as technical challenges.

The problems of interpretation can be appreciated by considering the chain of reactions possible for negative muons after stopping in a hydrogen target of density $\phi$ relative to liquid hydrogen $\left(\mathrm{LH}_{2}\right)$ [4]. Stopped muons immediately form ground state $\mu p$ atoms whose hyperfine states are populated in a statistical manner. The upper triplet spin state is rapidly depopulated in collisions with $\mathrm{H}_{2}$ molecules, and for densities $\phi \geq 0.01$ all muons reach the $\mu p$ singlet state well before $100 \mathrm{~ns}$. From there, muons can either decay with a rate close to $\lambda_{\mu}^{+} \equiv 1 / \tau_{\mu}^{+} \approx$ $0.455 \times 10^{6} \mathrm{~s}^{-1}$, or be captured via reaction (1) at the predicted rate $\Lambda_{S} \approx 710 \mathrm{~s}^{-1}$. Complications arise at higher densities, however, as $\mu p$ atoms increasingly collide with target $\mathrm{H}_{2}$ molecules to form $p \mu p$ molecules. The $p \mu p$ molecules are initially created in the ortho state at the 


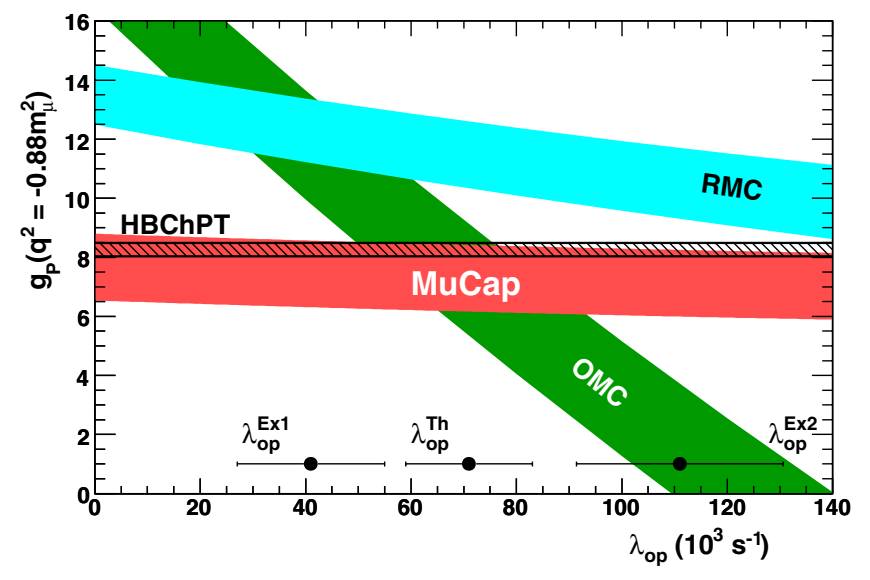

FIG. 1 (color online). Experimental and theoretical determinations of $g_{P}$, presented vs the ortho-para transition rate $\lambda_{\mathrm{op}}$ in the $p \mu p$ molecule. The most precise previous OMC experiment [10] and the RMC experiment [6] both depend significantly on the value of $\lambda_{\text {op }}$, which itself is poorly known due to mutually inconsistent experimental $\left(\lambda_{\mathrm{op}}^{\mathrm{Ex} 1}\right.$ [7], $\lambda_{\mathrm{op}}^{\mathrm{Ex}}$ [8]) and theoretical ( $\left.\lambda_{\mathrm{op}}^{\mathrm{Th}}[9]\right)$ results. In contrast, the MuCap result for $g_{P}$ is nearly independent of molecular effects.

density-dependent rate $\phi \lambda_{\text {of }}$, where $\lambda_{\text {of }} \approx 2.3 \times 10^{6} \mathrm{~s}^{-1}$, and then deexcite to the para state at rate $\lambda_{\mathrm{op}}$. The nuclear capture rates from the ortho and para states, $\Lambda_{\text {om }} \approx$ $506 \mathrm{~s}^{-1}$ and $\Lambda_{\mathrm{pm}} \approx 208 \mathrm{~s}^{-1}$ [3], are quite different from each other and from $\Lambda_{S}$, so knowledge of the relative populations of the $\mu p$ and $p \mu p$ states under any particular set of experimental conditions is crucial for a correct determination of $g_{P}$. Alas, $\lambda_{\text {op }}$ is poorly known [7-9]. This prevents a clear interpretation of the most precise OMC experiment [10], which was performed in $\mathrm{LH}_{2}$ where muon capture occurs predominantly in $p \mu p$ molecules. The RMC process is less sensitive to $\lambda_{\mathrm{op}}$, but the large molecular uncertainties make it difficult to draw firm conclusions from the RMC experiment [6], whose results initially suggested a nearly $50 \%$ higher value for $g_{P}$ than predicted.

Direct measurement of $\Lambda_{S}$ is technically difficult because process $(1)$ is rare (branching ratio $=0.16 \%$ ) and leads to an all-neutral final state. Moreover, target impurities and muon stops in detector walls must be scrupulously avoided, as negative muons preferentially and irreversibly transfer from $\mu p$ to heavier elements, and the nuclear muon capture rate increases roughly proportional to $Z^{4}$. The two previous muon capture experiments using lowdensity gas targets and neutron detectors obtained precisions in $\Lambda_{S}$ of $9 \%$ [11] and 13\% [12].

The MuCap experiment employs novel techniques to minimize or avoid many of the problems described above. The measurement is performed using hydrogen at density $\phi=(1.12 \pm 0.01) \times 10^{-2}$, where $p \mu p$ formation is slow and $96 \%$ of all captures proceed from the $\mu p$ singlet state. The significant background from muon stops in wall ma- terials, inherent when using a low-density target, is eliminated by reconstructing the muon stopping point in an active target consisting of a hydrogen time projection chamber (TPC). The capture rate is determined using the lifetime technique [10], that is, from the difference between the measured disappearance rate $\lambda_{\mu}^{-} \approx \lambda_{\mu}^{+}+\Lambda_{S}$ of negative muons in hydrogen and the $\mu^{+}$decay rate $\lambda_{\mu}^{+}$, where it is assumed that free $\mu^{-}$and $\mu^{+}$decay at identical rates according to the $C P T$ theorem.

The experiment was conducted in the $\pi \mathrm{E} 3$ beam line at the Paul Scherrer Institute, using $a \approx 20 \mathrm{kHz} d c$ muon beam tuned to a central momentum of $32.6 \mathrm{MeV} / c$. As illustrated in Fig. 2, incident muons first traverse a plastic scintillator $(\mu \mathrm{SC})$ and a multiwire proportional chamber ( $\mu \mathrm{PC}$ ), and then pass through a 0.5 -mm-thick hemispherical beryllium window to enter an aluminum pressure vessel filled with ultrapure, deuterium-depleted hydrogen gas at a pressure of $1.00 \mathrm{MPa}$ and at ambient room temperature. In the center of the vessel is the TPC (sensitive volume $15 \times$ $12 \times 28 \mathrm{~cm}^{3}$ ), which tracks incoming muon trajectories and thus enables the selection of muons that stop in the gas at least $15 \mathrm{~mm}$ away from chamber materials. Approximately $65 \%$ of the muons passing through the $\mu \mathrm{SC}$ stop within this fiducial volume. The ionization electrons produced by incoming muons drift downwards at velocity $5.5 \mathrm{~mm} / \mu \mathrm{s}$ in an applied field of $2 \mathrm{kV} / \mathrm{cm}$, towards a multiwire proportional chamber containing perpendicular anode and cathode wires. The anode plane consists of wires with $25 \mu \mathrm{m}$ diameter and $4 \mathrm{~mm}$ spacing, and a high voltage of $5.0 \mathrm{kV}$ across the $3.5 \mathrm{~mm}$ half-gaps achieves a gain of 60 in hydrogen.

The TPC is surrounded by two cylindrical wire chambers $(e \mathrm{PC} 1, e \mathrm{PC} 2)$, and by a hodoscope barrel $(e \mathrm{SC})$ consisting of 16 segments with two layers of 5-mm-thick

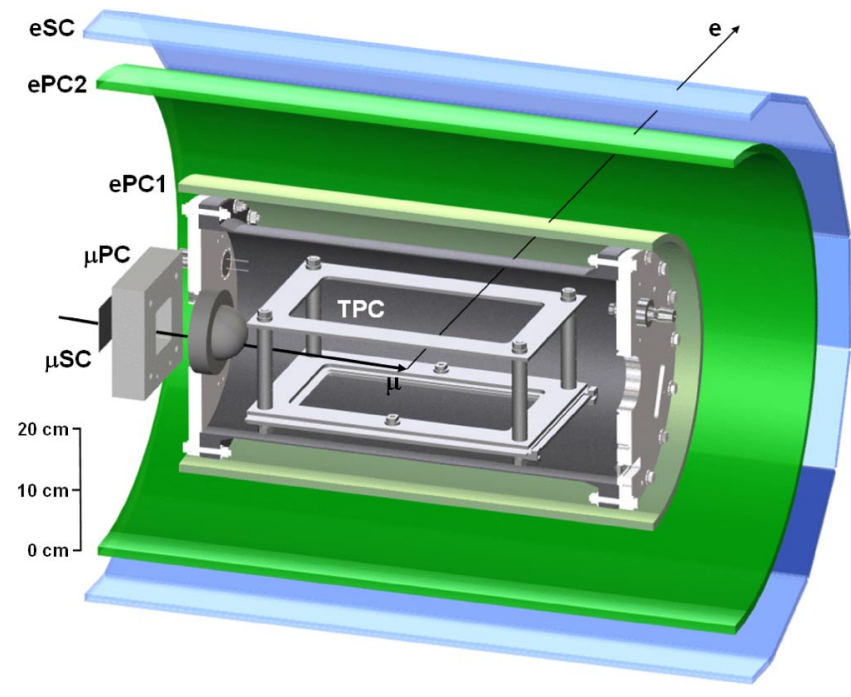

FIG. 2 (color online). Simplified cross-sectional diagram of the MuCap detector. The detector components are described in the text. 
plastic scintillator. This tracking system detects outgoing decay electrons with $3 \pi$ solid angle acceptance. All data are recorded in a triggerless, quasicontinuous mode to avoid deadtime distortions to the lifetime spectra. Custom-built time-to-digital converters (TDCs) digitize hit times for the TPC and the $e$ PCs. The muon and electron times $t_{\mu}$ and $t_{e}$ are established by the $\mu \mathrm{SC}$ and $e \mathrm{SC}$ detectors, and recorded in separate CAEN V767 TDC modules.

All TPC materials were carefully selected for high vacuum operation. Prior to the run, the TPC system was heated to $115^{\circ} \mathrm{C}$ under vacuum for several weeks to remove impurities. The system was filled with deuterium-depleted hydrogen through a palladium filter to remove impurities. During data taking, the gas was continuously circulated via an adsorption cryopump system and cleaned by cooled Zeolite filters [13], which achieved an equilibrium concentration (by number) of $c_{Z}<5 \times 10^{-8}$, as monitored by direct TPC detection of recoil nuclei from muon capture by impurities. Gas chromatography measurements established that the atomic concentration of nitrogen was below $10^{-8}$, and the post-run installation of a humidity sensor with $10^{-9}$ sensitivity into the gas circuit indicated that the primary contaminant was $\mathrm{H}_{2} \mathrm{O}$ outgassing from within the pressure vessel.

The isotopic purity of the hydrogen is critical. Muons preferentially transfer from $\mu p$ to $\mu d$ at the rate $\phi c_{d} \lambda_{p d}$, where $c_{d}$ is the deuterium concentration and $\lambda_{p d} \approx 1.4 \times$ $10^{10} \mathrm{~s}^{-1}$. Whereas $\mu p$ diffusion is on the order of $\mathrm{mm}, \mu d$ atoms can diffuse $\mathrm{cm}$-scale distances due to a RamsauerTownsend minimum in the $\mu d+p$ elastic scattering cross section. As a result, $\mu d$ atoms can drift sufficiently far away from the muon's original stopping point that the decay event will be rejected by the $\mu$-e vertex reconstruction cut in a time-dependent manner. In addition, $\mu d$ atoms can drift into surrounding materials and be captured there. Our target gas was produced via electrolysis of deuteriumdepleted water, and accelerator mass spectrometry measurements [14] determined that $c_{d}=(1.44 \pm 0.13) \times$ $10^{-6}$, roughly 100 times below deuterium's natural abundance. This result was independently confirmed from our data, by analyzing the observed losses of muon decay events as a function of the imposed $\mu-e$ vertex cut.

The time differences between muon arrivals and decay electron emissions, $\Delta t=t_{e}-t_{\mu}$, are histogrammed into lifetime spectra (Fig. 3). Only muons that are separated in time by $\pm 25 \mu$ s from other muon arrivals are accepted. While this condition cuts the usable statistics by $\approx 68 \%$, it is essential for avoiding systematic distortions to the background which can arise from ambiguities in resolving multiple muon tracks in the TPC, and it dramatically improves the signal-to-background ratio. As shown in Fig. 3, further background suppression can be achieved by performing a vertex cut on the impact parameter between each decay electron's trajectory and its parent

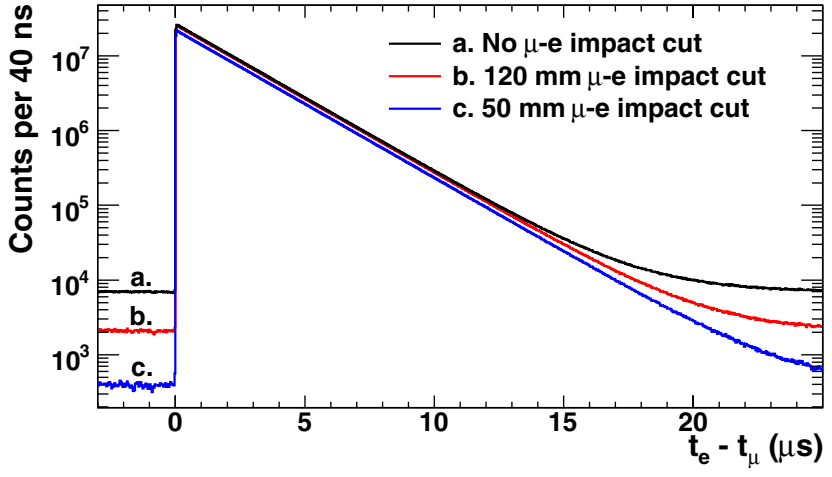

FIG. 3 (color online). Lifetime spectra of negative muons. The signal-to-background ratio improves with tighter cuts on the $\mu-e$ vertex.

muon's stopping point. In the final analysis we employ a loose impact parameter cut of $120 \mathrm{~mm}$ as an optimal compromise between the competing demands for a good signal-to-background ratio and minimization of losses due to $\mu d$ diffusion out of the cut volume.

We fit the $\mu^{-}$lifetime spectra with the simple exponential function $f(t)=N w \lambda e^{-\lambda t}+B$, where the free parameters are the number of reconstructed decay events $N$, the disappearance rate $\lambda$, and the accidental background level $B ; w$ is the fixed $40 \mathrm{~ns}$ histogram bin width. We studied an assortment of analysis conditions, including different time ranges (0.1-24 $\mu$ s is typical), fiducial cuts, and detector combinations, and typically obtained $\chi^{2} /$ dof $=0.95-1.02$ for 600 degrees of freedom.

In reality, the experimental $\mu^{-}$lifetime spectrum is not a pure exponential, but has a more complicated shape due to contributions from $p \mu p$ molecules and hydrogen gas impurities. However, these effects are sufficiently small that their perturbations $\Delta \lambda$ to the exponential decay rate $\lambda$ are linear and can be corrected sequentially. The main corrections to $\lambda$ were derived directly from experimental data, with some additional information from external measurements and literature. For residual $c_{Z}$ below a few times $10^{-6}$, the correction $\Delta \lambda_{Z}$ scales with the observed impurity capture yield per muon, $Y_{Z}$, as $\Delta \lambda_{Z}=Y_{Z}\left[\alpha_{N}\left(\frac{\Delta \lambda_{N}}{Y_{N}}\right)^{\text {calib }}+\right.$ $\left.\alpha_{O}\left(\frac{\Delta \lambda_{O}}{Y_{O}}\right)^{\text {calib }}\right]$. The observed yield $Y_{Z} \approx 11 \times 10^{-6}$ received contributions from nitrogen and humidity in weights of approximately $\alpha_{N}=0.05$ and $\alpha_{O}=0.95$. The factors $\left(\frac{\Delta \lambda}{Y}\right)^{\text {calib }}$ were empirically fixed by calibration runs involving $\mathrm{N}_{2}$ and $\mathrm{O}$ (in the form of $\mathrm{H}_{2} \mathrm{O}$ ) concentrations 50-1000 times above their values in the clean fill. We find $\Delta \lambda_{Z}=-19.2 \pm 5.0 \mathrm{~s}^{-1}$, where the error is dominated by a conservative estimate of the $\left(\frac{\Delta \lambda}{Y}\right)^{\text {calib }}$ value for $\mathrm{O}$, determined during our 2006 running period. The correction for deuterium-related diffusion effects, $\Delta \lambda_{d}=$ $-10.2 \pm 1.6 \mathrm{~s}^{-1}$, was obtained by a zero-extrapolation procedure using data from a run with a hydrogen filling of $c_{d}=(122 \pm 5) \times 10^{-6}$. 
The preceding corrections are summarized in Table I. There we also present four additional sources of uncertainty, including a conservative error of $5 \mathrm{~s}^{-1}$ that accounts for the spread in results observed for a variety of consistency studies, as performed by two independent analyses. To prevent bias, the master clock (accurate to $10^{-8}[15]$ ) was detuned by an offset which was concealed until the data analysis was complete.

The final result for the $\mu^{-}$disappearance rate in pure hydrogen, based on $N=1.6 \times 10^{9}$ fully tracked, pileupprotected decay events from our 2004 data set, is $\lambda_{\mu}^{-}=$ $455851.4 \pm 12.5_{\text {stat }} \pm 8.5_{\text {syst }} \mathrm{s}^{-1}$. As a consistency check, we also measured the $\mu^{+}$decay rate from $N=0.5 \times 10^{9}$ events to be $\lambda_{\mu}^{+}=455164 \pm 28 \mathrm{~s}^{-1}$, in agreement with the world average.

The observed $\mu^{-}$disappearance rate can be written as

$$
\lambda_{\mu}^{-}=\left(\lambda_{\mu}^{+}+\Delta \lambda_{\mu p}\right)+\Lambda_{S}+\Delta \Lambda_{p \mu p}
$$

Here $\Delta \lambda_{\mu p}=-12.3 \mathrm{~s}^{-1}$ describes a small reduction in the muon decay rate in the bound $\mu p$ system [16]. The term $\Delta \Lambda_{p \mu p}=-23.5 \pm 4.3 \pm 3.9 \mathrm{~s}^{-1}$ accounts for captures from $p \mu p$ molecules, and is calculated from the full $\mu^{-}$kinetics in pure hydrogen. Its error terms come from our estimates $\lambda_{\text {of }}=(2.3 \pm 0.5) \times 10^{6} \mathrm{~s}^{-1}$ and $\lambda_{\text {op }}=$ $(6.9 \pm 4.3) \times 10^{4} \mathrm{~s}^{-1}$, respectively, which cover most of the existing literature values. As muon capture from the $\mu p$ singlet component dominates both in $\mu p$ atoms and $p \mu p$ molecules, $\Delta \Lambda_{p \mu p}$ implicitly depends on $\Lambda_{S}$, which leads to a $3.2 \%$ loss in sensitivity when determining $\Lambda_{S}$ from Eq. (2). Using the new world average $\lambda_{\mu}^{+}=$ $455162.2 \pm 4.4 \mathrm{~s}^{-1}$ [15], we determine the rate of muon capture by the proton to be

$$
\Lambda_{S}^{\mathrm{MuCap}}=725.0 \pm 13.7_{\text {stat }} \pm 10.7_{\text {syst }} \mathrm{s}^{-1} .
$$

To compare with theory we consider the two recent nextto-next-to-leading-order calculations of $\Lambda_{S}, 687.4 \mathrm{~s}^{-1}$ [17] and $695 \mathrm{~s}^{-1}$ [18], here averaged to $691.2 \mathrm{~s}^{-1}$. Adding the very recently calculated radiative correction $\Delta_{R}=$ $19.4 \mathrm{~s}^{-1}$ [19] (increased from $\Delta_{R}=4.5 \mathrm{~s}^{-1}$ [20]) yields the value $\Lambda_{S}^{\mathrm{Th}}=710.6 \mathrm{~s}^{-1}$ and enables us to calculate

$$
g_{P}^{\mathrm{MuCap}}=g_{P}^{\mathrm{Th}}+\frac{\partial g_{P}}{\partial \Lambda_{S}}\left(\Lambda_{S}^{\mathrm{MuCap}}-\Lambda_{S}^{\mathrm{Th}}\right)=7.3 \pm 1.1
$$

where $g_{P}^{\mathrm{Th}}=8.26$ [3], $\frac{\partial g_{P}}{\partial \Lambda_{S}}=-0.065 \mathrm{~s}$ [5], and only the experimental uncertainty from Eq. (3) is propagated. The linear expansion in Eq. (4) is valid because $\Lambda_{S}^{\text {MuCap }}-\Lambda_{S}^{\text {Th }}$ is small, but should be refined once further theoretical work clarifies the present $1 \%$ difference between calculations $[17,18]$ and quantifies all sources of theoretical uncertainty at the subpercent level.
TABLE I. Systematic corrections and uncertainties applied to the observed $\mu^{-}$disappearance rate $\lambda$.

\begin{tabular}{lcc}
\hline \hline \multicolumn{1}{c}{ Source } & Correction $\left(\mathrm{s}^{-1}\right)$ & Uncertainty $\left(\mathrm{s}^{-1}\right)$ \\
\hline$Z>1$ impurities & -19.2 & 5.0 \\
$\mu d$ diffusion & -10.2 & 1.6 \\
$\mu p$ diffusion & -2.7 & 0.5 \\
$\mu+p$ scattering & & 3 \\
$\mu$ pileup veto efficiency & & 3 \\
Analysis methods & & 5 \\
Total & -32.1 & 8.5 \\
\hline \hline
\end{tabular}

The current information on $g_{P}$ is summarized in Fig. 1; the constraints [4] from the OMC experiment [10] are updated to reflect the larger $\Delta_{R}$ [19]. The situation before MuCap exhibited mutually inconsistent theoretical predictions and experimental determinations of both $g_{P}$ and $\lambda_{\mathrm{op}}$. The low gas density in MuCap renders our result relatively insensitive to $\lambda_{\mathrm{op}}$ and thus avoids most model dependence, enabling us to report the first precise, unambiguous determination of $g_{P}$. This experimental result agrees with present theory to within $1 \sigma$ and does not support a dramatic deviation from the chiral prediction as the RMC result originally had implied. Additional data are being collected with the aim of a more than twofold reduction of statistical and systematic uncertainties.

We are grateful to the technical staff of the collaborating institutions for their vital contributions to the experiment. Thanks are due to A. Adamczak, N. Bondar, L. Bonnet, R. M. Carey, P. T. Debevec, A. Dijksman, D. Fahrni, T. Ferguson, A. A. Fetisov, B. Gartner, J. Govaerts, F. J. Hartmann, A. Hofer, V.I. Jatsoura, V. Markushin, L. Meier, D. Michotte, C. J. G. Onderwater, J. Paley, S. Sadetsky, and P. A. Zolnierczuk for their contributions to the development of the experiment. This work was supported in part by the U.S. NSF, U.S. DOE and CRDF, PSI, the Russian Acad. Sci., and Russian grant No. NSH3057.2006.2. NCSA provided essential computing resources.

*Corresponding author.

[1] This estimate is dominated by the uncertainty in $\left|g_{A}(0)\right|=$ $1.2695 \pm 0.0029$, from W.-M. Yao et al. (Particle Data Group), J. Phys. G 33, 1 (2006).

[2] N. Kaiser, Phys. Rev. C 67, 027002 (2003).

[3] V. Bernard, L. Elouadrhiri, and U.-G. Meissner, J. Phys. G 28, R1 (2002). The original HBChPT derivation of $g_{P}$ is given in V. Bernard, N. Kaiser, and U.-G. Meissner, Phys. Rev. D 50, 6899 (1994).

[4] T. Gorringe and H.W. Fearing, Rev. Mod. Phys. 76, 31 (2003).

[5] J. Govaerts and J.-L. Lucio-Martinez, Nucl. Phys. A 678, 110 (2000).

[6] D. H. Wright et al., Phys. Rev. C 57, 373 (1998). 
[7] G. Bardin et al., Phys. Lett. B 104, 320 (1981).

[8] J. H. D. Clark et al., Phys. Rev. Lett. 96, 073401 (2006).

[9] D. D. Bakalov, M. P. Faifman, L. I. Ponomarev, and S. I. Vinitsky, Nucl. Phys. A384, 302 (1982).

[10] G. Bardin et al., Nucl. Phys. A352, 365 (1981).

[11] A. Alberigi Quaranta et al., Phys. Rev. 177, 2118 (1969).

[12] V. M. Bystritsky et al., Zh. Eksp. Teor. Fiz. 66, 43 (1974) [Sov. Phys. JETP 39, 19 ( 1974)].

[13] V. A. Ganzha et al., arXiv:0705.1473v1 [Nucl. Instrum. Methods Phys. Res., Sect. A (to be published)].

[14] H. Synal, M. Stocker, and M. Suter, Nucl. Instrum. Methods Phys. Res., Sect. B 259, 7 (2007).
[15] D. B. Chitwood et al. (MuLan Collaboration), Phys. Rev. Lett. 99, 032001 (2007).

[16] H. Überall, Phys. Rev. 119, 365 (1960); H. C. Von Baeyer and D. Leiter, Phys. Rev. A 19, 1371 (1979).

[17] V. Bernard, T. R. Hemmert, and U.-G. Meissner, Nucl. Phys. A686, 290 (2001).

[18] S. Ando, F. Myhrer, and K. Kubodera, Phys. Rev. C 63, 015203 (2000).

[19] A. Czarnecki, W. J. Marciano, and A. Sirlin Phys. Rev. Lett. 99, 032003 (2007).

[20] M. R. Goldman, Nucl. Phys. B49, 621 (1972). 